\title{
DOENÇA DE NIEMANN-PICK TIPO C DE INÍCIO TARDIO: RELATO DE CASO
}

\section{LATE-ONSET NIEMANN-PICK DISEASE TYPE C: CASE REPORT}

\author{
Enio Soares Dos Santos Junior*, Custódio Camargo Fernandes, Diogo Fraxino \\ De Almeida.
}

CESUMAR - Centro Universitário de Maringá, Maringá, PR, Brasil. *eeniojunior@gmail.com

\section{RESUMO}

A doença de Niemann Pick Tipo C (NP-C) é um distúrbio genético raro decorrente de um erro inato do metabolismo, o qual repercute no metabolismo do colesterol não esterificado, o qual se torna neurodegenerativo. O objetivo deste resumo é apresentar um caso de paciente com doença de NP-C de início tardio, com suas devidas particularidades e dessa forma, discutir as características de sua patologia e contribuir com conhecimento acerca da doença. Estudo foi aprovado pelo comitê de ética da UniCesumar (Protocolo n-3.582.503). Relato do caso: Paciente masculino, 59 anos, há cerca de 7 anos começou a apresentar dificuldade de coordenação motora e ataxia. Nesse período evoluiu com disartria e problemas cognitivos relacionados a memória recente. Foi inicialmente diagnosticado com Mal de Parkinson. Posteriormente, devido associação de ataxia com distúrbio da motilidade ocular vertical foi suspeitada a possibilidade de NP-C. Foi realizada biópsia de pele com teste de Filipin que foi negativa e dosagem da quitotriosidade normal. $O$ biomarcador Lyso SM-509 teve resultado aumentado e o teste genético confirmou o diagnóstico de NP-C com mutação NPC1. Foi iniciado tratamento com Miglustate $200 \mathrm{mg}$ e aconselhamento genético. A doença de NP-C é uma condição genética autossômica ressessiva. Abordamos uma forma tardia da doença que se desenvolveu apenas os 52 anos de idade, onde seu aparecimento é tão atípico, que o diagnóstico se torna complexo, podendo ser confundido com outras doenças. A ataxia cerebelar e a oftalmoplegia supranuclear são manifestações comuns de serem encontradas nas formas infantil e adulta, variando em progressão, sendo esta mais lenta nos adultos. As manifestações podem variar, como disartria, disfagia, comprometimento cognitivo e outros distúrbios do movimento, como tremores e distonia. Esses sintomas estavam presentes no caso descrito, mas que diante da idade de apresentação, compartilham quadro clínico semelhante de outras doenças mais prevalentes na faixa etária do paciente, como a doença de Parkinson, levando a esse diagnóstico inicialmente. O diagnóstico da NP-C se dá pelos sintomas característicos da doença juntamente com testes bioquímicos e genéticos. A biópsia de pele para o teste de Filipin pode ser usada em casos inconclusivos. Sendo assim, diante da variedade de sintomas possíveis para a NP-C, um paciente em uma faixa etária atípica para a doença, o diagnóstico torna-se um desafio. A NP-C possui tratamento, mas é incurável. Estudos comprovaram que o Miglustate reduz a progressão dos sintomas neurológicos associados a NP-C. Desta forma, conclui- 
se que a forma tardia de NP-C é difícil de ser reconhecida, pois toda a sua gama de sinais e sintomas sinalizam a favor de outras doenças mais comuns na faixa etária. Portanto, é importante reconhecer a oftalmoplegia supranuclear e ataxia, principalmente quando combinadas, NP-C sempre deve ser incluída no diagnóstico diferencial.

Palavras-chave: Ataxia. Distonia. Estudo de caso. Mal de Parkinson. Oftalmoplegia. 